\title{
NEWSdm: Nuclear Emulsions for WIMP Search with directional measurement
}

\author{
A. Di Crescenzo ${ }^{1,2, a}$ on behalf of the NEWSdm Collaboration \\ ${ }^{1}$ Dipartimento di Fisica dell'Università Federico II di Napoli \\ ${ }^{2}$ INFN Sezione di Napoli
}

\begin{abstract}
Direct Dark Matter searches are nowadays one of the most exciting research topics. Several experimental efforts are concentrated on the development, construction, and operation of detectors looking for the scattering of target nuclei with Weakly Interactive Massive Particles (WIMPs). The measurement of the direction of WIMP-induced nuclear recoils is a challenging strategy to extend dark matter searches beyond the neutrino floor and provide an unambiguous signature of the detection of Galactic dark matter. Current directional experiments are based on the use of gas TPC whose sensitivity is strongly limited by the small achievable detector mass. We present an innovative directional experiment based on the use of a solid target made by newly developed nuclear emulsions and read-out systems reaching a position resolution of the order of $10 \mathrm{~nm}$.
\end{abstract}

\section{Introduction}

Current experimental efforts in the field of direct Dark Matter (DM) searches are devoted to the search for rare interactions between galactic halo WIMPs and nuclei in a detector on Earth. As dark matter detectors are rapidly improving in sensitivity, they will encounter the neutrino background, at which point Solar, atmospheric, and diffuse supernova neutrinos can mimic the dark matter signal. Neutrinos are therefore the ultimate background for WIMP direct detection searches as they cannot be shielded against and produce recoils with similar rates and energy spectra. Moreover, the recent controversy in the low-mass WIMP region $\mathrm{O}\left(10 \mathrm{GeV} / \mathrm{c}^{2}\right)$, where some dark matter hints are inconsistent with null results, highlights the need for additional discrimination power between WIMP events and backgrounds in order to clearly authenticate a genuine WIMP signal.

New generation detectors capable of measuring the direction of a nuclear recoil track resulting from the elastic scattering of a target nucleus by an incoming WIMP (Weakly Interacting Massive Particle), would provides a route towards the discrimination of neutrino background and the unambiguous identification of WIMPs as being responsible for the galactic dark matter.

From the experimental point of view several approaches have been proposed. The more diffuse strategy is based on the use of low pressure gaseous Time Projection Chambers (TPCs) [1]. Experiments based on this technology have provided the first directional limits in the Spin-Dependent (SD) case and the collaborations aim at developing larger detectors to be operated in a medium term time scale. Nevertheless this technology is hardly scalable to very large detectors masses needed to reach a good

\footnotetext{
a e-mail: dicrescenzo@na.infn.it
} 
sensitivity to the Spin-Independent (SI) case.

The use of a solid target for directional searches would overcome the mass limitation of gaseous TPC approach thus allowing to reach an high sensitivity in the low cross section sectors of the SI case. Nevertheless, in a solid medium, the track of the WIMP-scattered nuclear recoil will have a path length of the order of a few hundred nanometers, much shorter than in the case of a gaseous target where the recoil length is expected to be of the order of a few millimeters. A detector with high tracking resolution is therefore needed.

\section{The NEWSdm experiment}

The approach proposed by the NEWSdm Collaboration [2] consists in the use of a nuclear emulsionbased detector acting both as target and as tracking device. The project foresees the employment of a novel emulsion technology called Nano Imaging Trackers (NIT) [3, 4] featuring a position resolution of an order of magnitude higher than that of the emulsion currently used in the OPERA experiment [5]. The detector is conceived as a bulk of NIT surrounded by a shield to reduce the external background. The detector is then placed on an equatorial telescope in order to absorb the Earth rotation, thus keeping fixed the detector orientation with respect to the incoming apparent WIMP flux. The angular distribution of the WIMP-scattered nuclei is therefore expected to be strongly anisotropic with a peak centered in the forward direction.

\subsection{NIT: Nano Imaging Trackers}

Nuclear emulsions are made of silver halide crystals embedded in a gelatine matrix. When ionizing particles pass through it, some of the halide crystals are modified in such a way that they are turned into grains of silver when a developing process is performed. The three-dimensional trajectory of passing through particles can be reconstructed with an optical microscope by connecting all the silver grains.

NIT are a novel type of emulsion films with grain diameters down to a few tens of nm, one order of magnitude smaller than conventional ones. They have a linear density of crystals of about 11 crystals $/ \mu \mathrm{m}[3]$ and they are made by the following nuclei (mass fraction in \%): $\mathrm{H}(1.6), \mathrm{C}(10.1), \mathrm{N}$ (2.7), $\mathrm{O}$ (7.4), S (0.3), $\mathrm{Br}(32.0), \mathrm{Ag}(44.0), \mathrm{I}(1.9)$. The total density is $3.2 \mathrm{~g} / \mathrm{cm}^{3}$. They make the reconstruction of trajectories with path lengths shorter than $100 \mathrm{~nm}$ possible, if analyzed by means of microscopes with enough resolution.

The presence in the emulsion gel of lighter nuclei such as carbon, oxygen and nitrogen, in addition to the heavier nuclei of silver and bromine, is a key feature of the NEWS project, resulting in a good sensitivity to WIMPs with both light and heavy masses.

\subsection{Read-out system}

In the NEWS experiment the expected WIMP signal will consist of short-path, anisotropically distributed, nuclear recoils over an isotropically distributed background. The search for signal candidates requires the scanning of the whole emulsion volume. The read-out system has therefore to fulfill two main requirements: a fast, completely automated, scanning system is needed to analyse the target volume over a time scale comparable with the exposure; the spatial resolution has to be improved by more than one order of magnitude compared to that achieved with standard emulsion films, reaching the challenging value of a few tens of nanometers, in order to ensure high efficiency and purity in the selection of signal candidates. 
The analysis of NIT emulsions is performed with a two-step approach: a fast scanning with a state-ofthe-art resolution for the signal preselection followed by a pin-point check of preselected candidates with unprecedented nanometric resolution to further enhance the signal to noise ratio and perform very accurate measurements of the range and the recoil direction. In the first phase a fast scanning is performed by means of an improved version of the optical microscope used for the scanning of the OPERA films [7, 9]. In the last years an R\&D program aimed at improving the ESS performances was carried by INFN groups, leading to prototypes with resolution improved by one order of magnitude, achieving a speed of almost $200 \mathrm{~cm}^{2} / \mathrm{h}$ [8]. A new system is being developed in Japan (the Super-Ultra Track Selector), aiming at increasing the scanning speed up to $5000 \mathrm{~cm}^{2} / \mathrm{h}$.

The starting point of the emulsion scanning is the image analysis to collect clusters made of dark grains at several depths across the emulsion plate thickness. Given the intrinsic resolution of the optical microscope $(\sim 200 \mathrm{~nm})$, the sequence of several grains making a track of a few hundred nanometers, appears as a single cluster. Therefore, the key element to distinguish clusters made of several grains from clusters made of a single grain produced by thermal excitation $(f \circ g)$ is the analysis of their shape. A cluster made of several grains indeed tends to have an elliptical shape with the major axis along the direction of the trajectory, while a cluster produced by a single grain tends to have a spherical shape. In order to simulate the effect of a WIMP-induced nuclear recoil and to measure the efficiency and the resolution of the new optical prototype, a test beam with low velocity ions was performed. $\mathrm{Kr}$ ion beam with energies of 200 and $400 \mathrm{keV} \mathrm{[10]} \mathrm{and} \mathrm{a} \mathrm{C} \mathrm{ion} \mathrm{beam} \mathrm{with} \mathrm{energies} \mathrm{of} \mathrm{60,} 80$ and $100 \mathrm{keV}$ were used.

When analysed with the optical microscope, submicrometric tracks produced by $\mathrm{Kr}$ and $\mathrm{C}$ ions appear as shown in Fig. 1(a). Although silver grains belonging to the tracks are not distinguishable and appear as a single cluster, the elongated form of the cluster is clearly visible [11]. An elliptical fit of the cluster shape allows a clear separation between fog grains and signal tracks: the latter ones are expected to have ellipticity larger than a given threshold, typically 1.25 or higher.

In order to evaluate the intrinsic angular resolution of the scanning system we analysed an emulsion sample exposed to a $2.8 \mathrm{MeV}$ neutron beam at the Fusion Neutronics Source (FNS) of the Japan Atomic Energy Agency (JAEA). An intrinsic angular resolution of $230 \mathrm{mrad}$ was achieved with fully automated scanning systems, by far the best resolution achieved with direction sensitive detectors in this energy range. The simulation shows that this result is compatible with the measurement reported above when the scattering contribution is included.

The second phase is performed exploiting the resonance effect occurring when nanometric metal grains are dispersed in a dielectric medium [12]. The polarization dependence of the resonance frequencies strongly reflects the shape anisotropy and can be used to infer the presence of non-spherical nanometric silver grains. NEWS will use this technology to retrieve track information in NIT emulsions beyond the optical resolution. Images of the same cluster taken with different polarization angles will show a displacement of the position of its barycenter. The analysis of the displacements allows to distinguish clusters made of a single grain from those made of two (or more) grains.

The evaluation of the position accuracy was performed by analysing images of single grains. The unprecedented accuracy of about $10 \mathrm{~nm}$ can be achieved in both coordinates (Fig. 1(b)).

\section{Background}

Background sources for dark matter searches are $\alpha$ and $\beta$ particles, $\gamma$-rays and neutron induced recoils, while NIT are essentially not sensitive to minimum ionizing particles (MIP).

The main sources of $\alpha$-particles are $\mathrm{U}$ and Th radioactive chains and Radon. The $\alpha$-particles produced in those processes have energies of the order of $\mathrm{MeV}$ and their range in emulsion is of the order of tens 


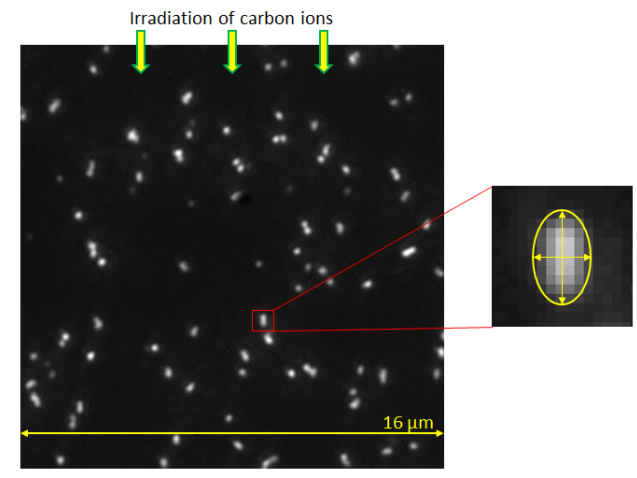

(a)

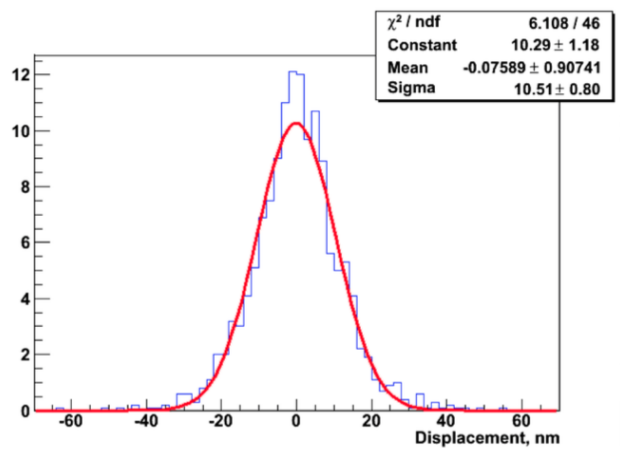

(b)

Figure 1. 1(a) Kr ions implanted on NIT films. The image is taken with an optical microscope. The selection of candidate tracks is based on the elliptic fit of the clusters. 1(b) Position accuracy of about $10 \mathrm{~nm}$ with the resonant light scattering.

of microns, by far longer than WIMP-induced nuclear recoils. $\alpha$-particles can therefore be identified and discarded in the emulsions by an upper cut on the track length.

The $\beta$-rays produced in ${ }^{14} \mathrm{C}$ decay constitute a non-negligible contribution to the total background budget. This kind of background is anyway less critical for NIT emulsion with respect to other sources: electrons can be rejected by properly regulating the emulsion response, in terms of number of sensitized crystals per unit path length (i.e. the sensitivity), through a chemical treatment of the emulsion itself. Moreover possible improvements in the rejection power can be achieved exploiting the response of $\beta$-rays to the polarized light scattering or performing a cryogenic exposure and by exploiting the phonon effect.

Neutron induced recoils are the main background source because they are not distinguishable from the expected WIMP signal, except for the isotropic angular distribution and for the typical track length, largely exceeding the range expected for WIMP-induced recoils. Three types of neutron sources affect underground experiments: radiogenic neutrons in the $\mathrm{MeV}$ range produced in $(\alpha, \mathrm{n})$ and spontaneous fission reactions in the detector due to its intrinsic radioactive contaminants, cosmogenic neutrons with energy spectrum extending to $\mathrm{GeV}$ energies induced by muons penetrating underground through the rock, neutrons induced by environmental radioactivity.

While the external neutron flux can be reduced to a reasonable level with an appropriate shielding, the intrinsic emulsion radioactivity would be responsible of an irreducible neutron yield through $(\alpha$, n) and ${ }^{238} \mathrm{U}$ spontaneous fission reaction. In order to estimate this contribution, the activities of $U$ and $\mathrm{Th}$ in the emulsion components has been measured with the Inductively Coupled Plasma Mass Spectrometry (ICP-MS) and with the $\gamma$-spectrometry. By using a dedicated MC simulation based on the SOURCES code [13], the corresponding neutron yield has been estimated to be of the order of $1.2 \pm 0.4 \mathrm{n} / \mathrm{yr} / \mathrm{kg}$. The neutron energy spectrum, as calculated with SOURCES, was then used as the input for a GEANT4-based simulation in order to estimate the fraction of neutrons interacting in the emulsion and laying in the signal region $(100 \mathrm{~nm}<\mathrm{L}<1 \mu \mathrm{m})$. The detectable neutron-induced background would be $0.02 \div 0.03$ per year per kilogram [14]. 


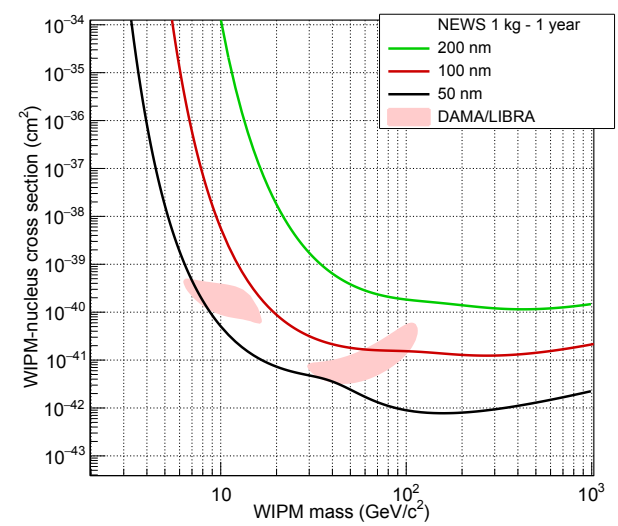

Figure 2. The $90 \%$ C.L. upper limits for a NIT detector with an exposure of $1 \mathrm{~kg} \times$ year, a threshold ranging from $200 \mathrm{~nm}$ down to $50 \mathrm{~nm}$, in the zero background hypothesis. The directionality information is not included.

\section{Experiment time-scale and sensitivity}

We plan to perform the first exposure with a target mass of $1 \mathrm{~kg}$ and the corresponding analysis of the data on a time scale of five years. The first year of the project will be devoted to the realization of a pilot exposure of $10 \mathrm{gr}$ in order to confirm the estimations of the overall background budget of the experiment. The second and third year of the project will be devoted to the construction of the infrastructures, the production of the emulsion target and of the detector shield. The exposure of 1 $\mathrm{Kg}$ detector is foreseen by the beginning of 2020 and will last one year. We plan to get first analysis results by the end of 2021 .

In Fig. 2 the 90\% C.L. upper limit, in case of null observation for an exposure of $1 \mathrm{~kg} \times$ year of NIT emulsions, with a minimum detectable track length ranging from $200 \mathrm{~nm}$ down to $50 \mathrm{~nm}$ and in the hypothesis of zero background, is shown. Even not including the directionality discrimination of the signal and assuming to reach a negligible background level, such an experiment would cover a large part of the parameter space indicated by the DAMA/LIBRA results with a small $(1 \mathrm{~kg})$ detector mass, using a powerful and complementary approach.

\section{Conclusions}

NEWS is meant to be the first experiment with a solid target for directional dark matter searches: the use of a nuclear emulsion based detector, acting both as target and tracking device, would allow to extend dark matter searches beyond the neutrino floor and provide an unambiguous signature of the detection of Galactic dark matter.

The novel emulsion technology, based on the use of nuclear emulsion with nanometric AgBr crystals (NIT), makes it possible to record the sub-micrometric tracks produced by the WIMP scattering off a target nucleus. The presence, in the emulsion components, of light and heavy nuclei results in an enhanced sensitivity to both light and heavy WIMP masses.

The final signal confirmation is obtained with powerful optical microscope equipped with a light polarizer: exploiting the different response of non spherical grain clusters to different polarization 
angles, the unprecedented spatial resolution of $10 \mathrm{~nm}$ is obtained. This resolution allows to resolve grains belonging to a few hundred of nanometer long tracks thus providing the

final signal confirmation with a very high signal to noise ratio.

We plan to perform a pilot experiment with a $1 \mathrm{~kg}$ mass target on a time scale of five years: even using a rather small detector mass we would be able to explore the region indicated by the DAMA experiment with a powerful and complementary approach.

The actual intrinsic radioactive level allows to scale the target mass and exposure time up to one order of magnitude. A careful selection of the emulsion components and a better control of their production could further increase the radiopurity, thus allowing larger detector mass. The reduction of the fog density and further developments of the optical microscopy with polarized light would allow to reduce the detection threshold down to $50 \mathrm{~nm}$.

Improvements both in the mechanics (use of piezoelectric-driven objective) and in the image acquisition (use of multiple image sensors) envisage the possibility to analyse with such a resolution a volume of $100 \mathrm{~kg}$ or larger.

\section{References}

[1] J.B.R. Battat et al., Phys. Rep 662 (2016) 1

[2] A. Alexandrov et al. (NEWS Collaboration), arXiv:1604.04199, LNGS-LOI 48/15

[3] M. Natsume et al., Nucl. Instr. Meth. A575 (2007) 439

[4] T. Naka et al., Nucl. Instrum. Meth. A718 (2013) 519

[5] A. Agafonova et al. (OPERA Collaboration), JINST 4 (2009) P04018.

[6] B. Morgan, A.M. Green and N.J.C. Spooner, Phys. Rev. D 71 (2005) 103507

[7] N. Armenise et al., Nucl. Instr. Meth. A551 (2005) 261

L. Arrabito et al., Nucl. Instr. Meth. A568 (2006) 578

[8] A. Alexandrov, V. Tioukov, M. Vladymyrov, JINST 9 (2014) C02034

[9] K. Morishima and T. Nakano, JINST 5 (2010) P04011

S. Aoki et al., Nucl. Instrum. Meth. B 51 (1990) 466

[10] T. Naka et al., EAS Publ. Ser. 53 (2012) 51

[11] M. Kimura and T. Naka, Nucl. Instrum. Meth. A680 (2012) 12

[12] H. Tamaru et al., Applied Phys. Lett. 80 (2002) 1826

[13] W.B. Wilson et al., LA-13639-MS, Los Alamos (1999)

[14] A. Alexandrov et al., Astropart. Phys. 80 (2016) 16 